\title{
Linear Wakefield Expression For Bi-Gaussian Drive Bunches
}

\author{
W. Lu, C. Huang, M.M. Zhou, C.E. Clayton, D.K. Johnson, C. Joshi, K.A. Marsh, \\ and W.B. Mori \\ University of California Los Angeles, CA 90095 \\ S. Deng, E. Oz, T. Katsouleas \\ University of Southern California, Los Angeles, CA 90089 \\ C.D. Barnes, C. O’Connell, F.J. Decker, P. Emma, R. Iverson, P. Krejcik, D. Walz \\ Stanford Linear Accelerator Center, Menlo Park CA 94275
}

\begin{abstract}
An expression for the maximum accelerating field in the narrow bunch limit $\left(\mathrm{k}_{\mathrm{p}} \sigma_{\mathrm{r}}<<1\right)$ for a bi-Gaussian drive beam is derived from linear plasma wakefield theory [1]. The optimal plasma density for a given beam $\left(\mathrm{N}, \sigma_{\mathrm{z}}, \sigma_{\mathrm{r}}\right)$ is found to be $k_{p} \sigma_{z}=\sqrt{2}$ in the narrow beam limit. We also show that the previously obtained scaling law [2,3] in which the wake amplitude increases with the inverse square of the bunch length must be modified by a weak logarithmic function of the beam spot size $\sigma_{r}$.
\end{abstract}

\section{INTRODUCTION}

This is a brief note whose purpose is to clarify some confusion in the literature regarding the prediction of linear theory for the wakes produced by narrow particle beam bunches. By narrow, we mean the beam's spot size is small compared to the collisionless skin depth. In linear theory there is not difference in the wake amplitude between electron and positron bunches (although there is a change in sign in the wake). We concentrate on bi-Gaussian shaped bunches. In a manuscript under preparation we give more details on the theory and discuss the validity of the linear predictions as the bunch charge is increased.

\section{LINEAR WAKEFIELD EXPRESSION FOR BI-GAUSSIAN DRIVE BUNCHES}

Starting from the well-known Green's function solution [1] for the plasma response to an arbitrary relativistic charge bunch of the form $\rho_{b}=\rho_{\perp}(r) * \rho_{\|}(\xi)(\xi=z-c t)$, we 
get the wake field at a position along the axis where $\xi<<-\sigma_{z}$ for a Gaussian longitudinal profile $\rho_{\|}(\xi)=q n_{b} e^{\frac{-\xi^{2}}{2 \sigma_{z}^{2}}}$ :

$$
E_{z}(0, \xi)=\left\{\sqrt{2 \pi}(q / e)\left(m c \omega_{p} / e\right)\left(n_{b} / n_{p}\right)\left(k_{p} \sigma_{z} e^{-\frac{k_{p}^{2} \sigma_{z}^{2}}{2}}\right) * R(0)\right\} * \operatorname{Cos}\left(k_{p} \xi\right)
$$

where

$$
R(0)=k_{p}^{2} \int_{0}^{\infty} r^{\prime} d r^{\prime} \rho_{\perp}\left(r^{\prime}\right) K_{0}\left(k_{p} r^{\prime}\right)
$$

and $\mathrm{K}_{0}$ is the zero-order modified Bessel function.

The expression for $R(0)$ for a flat top beam of radius $a$ with $\rho_{\perp}(r)=1$ for $0<r<a$ and 0 for $r>a$ is:

$$
R(0)=k_{p}^{2} \int_{0}^{a} r^{\prime} d r^{\prime} K_{0}\left(k_{p} r^{\prime}\right)=1-k_{p} a K_{1}\left(k_{p} a\right)
$$

which was given and was plotted numerically in [1].

The corresponding expression for $\mathrm{R}(0)$ for Gaussian bunches with $\rho_{\perp}(r)=e^{\frac{-r^{2}}{2 \sigma_{r}^{2}}}$ is:

$R(0)=\left(\frac{k_{p}^{2} \sigma_{r}^{2}}{2}\right)\left(e^{\frac{k_{p}^{2} \sigma_{r}^{2}}{2}}\right) \Gamma\left(0, \frac{k_{p}^{2} \sigma_{r}^{2}}{2}\right)$

where $\Gamma(\alpha, \beta)=\int_{\beta}^{\infty} t^{\alpha-1} e^{-t} d t$.

In the narrow beam limit ( $k_{p} a<<1$ or $k_{p} \sigma_{r}<<1$ ), eq.(3) and eq.(4) can be expanded asymptotically as :

$R(0)=\frac{k_{p}^{2} a^{2}}{2}\left(0.6159-\ln \left(k_{p} a\right)\right)$

and

$$
R(0)=k_{p}^{2} \sigma_{r}^{2}\left(0.05797-\ln \left(k_{p} \sigma_{r}\right)\right)
$$

In fig. 1 , we plot the ratio of (3) and (4) for $a=\sqrt{2} \sigma_{r}$ such that the peak density $n_{b}$ ( $n_{b}=\frac{N}{(2 \pi)^{\frac{3}{2}} \sigma_{z} \sigma_{r}^{2}}$ for Gaussian and $n_{b}=\frac{N}{(2 \pi)^{\frac{1}{2}} \sigma_{z}\left(\pi a^{2}\right)}$ for flat top transverse profiles) is the same for both profiles with the same number of particles $\mathrm{N}$. 


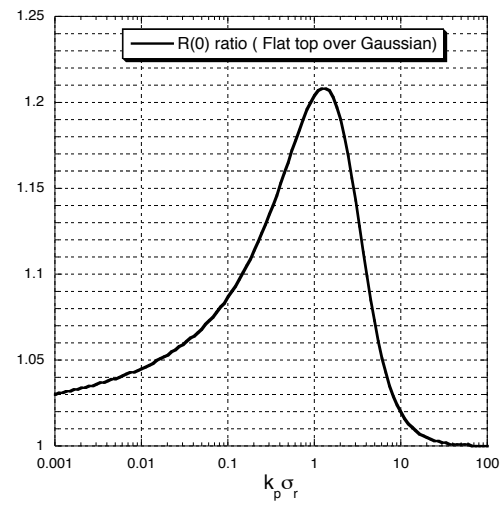

(a)

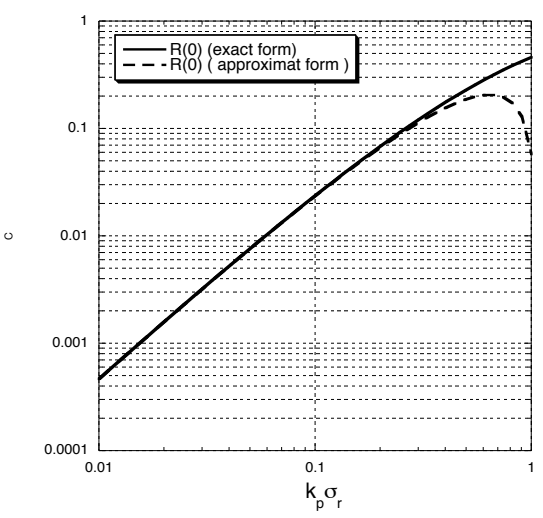

(b)

FIGURE 1. (a) The ratio between the $R(0)$ for flat top and Gaussian profiles.(b) Comparison between the exact form and asymptotic form for $R(0)$ for a Gaussian profile.

From figure (1a) we see that the $\mathrm{R}(0)$ for the two profiles differ by at most $20 \%$ near $k_{p} \sigma_{z} \approx 1.35$ and are nearly identical for $k_{p} \sigma_{z}<<1$ or $k_{p} \sigma_{z}>>1$. In figure (1b), for a transverse Gaussian profile, we plot both the full expression for $\mathrm{R}(0)$ (eq.4) and the 
asymptotic expression for small $\mathrm{k}_{\mathrm{p}} \sigma_{\mathrm{r}}$ (eq.6). This shows that the asymptotic expression is extremely accurate for $\mathrm{k}_{\mathrm{p}} \sigma_{\mathrm{r}}<0.5$.

Combining eqs.(1) and (4), we obtain an expression for the wake amplitude for a bi-Gaussian shaped drive beam:

$E_{z a}=\sqrt{2 \pi}\left(\frac{m c \omega_{p}}{e}\right)\left\{\left(\frac{q}{e}\right)\left(\frac{n_{b}}{n_{p}}\right)\left(k_{p} \sigma_{z} e^{-\frac{k_{p}^{2} \sigma_{z}^{2}}{2}}\right)\left(\frac{k_{p}^{2} \sigma_{r}^{2}}{2}\right)\left(e^{\frac{k_{p}^{2} \sigma_{r}^{2}}{2}}\right) \Gamma\left(0, \frac{k_{p}^{2} \sigma_{r}^{2}}{2}\right)\right\}$

This can be rewritten in terms of the total particle number $N=(2 \pi)^{\frac{3}{2}} \sigma_{r}^{2} \sigma_{z} n_{b}$ :

$E_{z a}=q N k_{p}^{2}\left\{\left(e^{-\frac{k_{p}^{2} \sigma_{z}^{2}}{2}}\right)\left(e^{\frac{k_{p}^{2} \sigma_{r}^{2}}{2}}\right) \Gamma\left(0, \frac{k_{p}^{2} \sigma_{r}^{2}}{2}\right)\right\}$

This explicitly shows the $k_{p}^{2}$ dependence of the wake amplitude for a beam with fixed $N, k_{p} \sigma_{z}$ and $k_{p} \sigma_{r}$.

\section{THE OPTIMAL WAKE CONDITION AND THE LINEAR SCALING LAW}

To get the optimal wake field amplitude for a beam with given $N, \sigma_{z}$ and $\sigma_{r}$, we rewrite eq.(8) as:

$E_{z a}=\frac{q N}{\sigma_{r} \sigma_{z}}\left\{\left(k_{p} \sigma_{z}\right)\left(e^{-\frac{k_{p}^{2} \sigma_{z}^{2}}{2}}\right)\left(k_{p} \sigma_{r}\right)\left(e^{\frac{k_{p}^{2} \sigma_{r}^{2}}{2}}\right) \Gamma\left(0, \frac{k_{p}^{2} \sigma_{r}^{2}}{2}\right)\right\}=\frac{q N}{\sigma_{r} \sigma_{z}} \Lambda\left(k_{p} \sigma_{z}, k_{p} \sigma_{r}\right)$

where $\Lambda\left(k_{p} \sigma_{z}, k_{p} \sigma_{r}\right)$ can also be viewed as a function of $\mathrm{k}_{\mathrm{p}} \sigma_{\mathrm{z}}$ and $\mathrm{r}_{\mathrm{a}} \equiv \sigma_{\mathrm{r}} / \sigma_{\mathrm{z}}$ and $\mathrm{r}_{\mathrm{a}}$ is defined as the beam's aspect ratio. Next, we maximize this expression for fixed particle number $\mathrm{N}, \sigma_{z}$, and $\sigma_{r}$, i.e., we find the optimal plasma density. Although the optimal $k_{p}$ depends on both $\sigma_{z}$ and $\sigma_{r}$, the maximum value for $\Lambda$ depends only on the aspect ratio $r_{a}$. So the maximum wake amplitude can be written as:

$$
E_{z M}=\frac{q N}{\sigma_{r} \sigma_{z}} \Theta\left(\frac{\sigma_{r}}{\sigma_{z}}\right)
$$




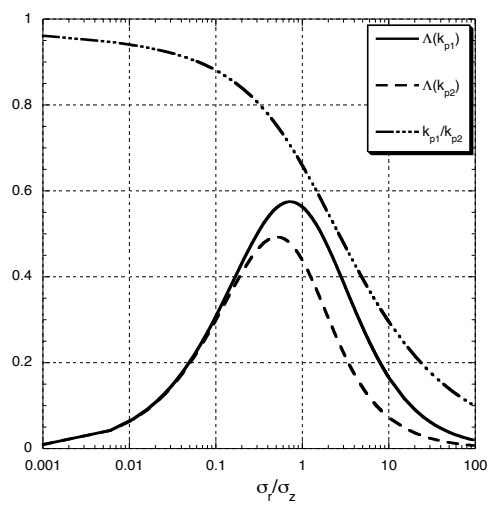

FIGURE 2. Plots of $\Lambda\left(k_{p 1} \sigma_{z}, k_{p 1} \sigma_{r}\right), \Lambda\left(k_{p 2} \sigma_{z}, k_{p 2} \sigma_{r}\right)$ and $k_{p 1} / k_{p 2}$

In Fig.2, we plot the function $\Lambda\left(k_{p 1} \sigma_{z}, k_{p 1} \sigma_{r}\right)$, where $k_{p 1}$ is the optimal $k_{p}\left(\Lambda\left(k_{p 1} \sigma_{z}, k_{p 1} \sigma_{r}\right) \equiv \Theta\left(\frac{\sigma_{r}}{\sigma_{z}}\right)\right)$ and the function $\Lambda\left(k_{p 2} \sigma_{z}, k_{p 2} \sigma_{r}\right)$, where $k_{p 2}=\sqrt{2} / \sigma_{z}$. It can clearly be seen that they agree very well as long as $\sigma_{r} / \sigma_{z}<0.1$. We also notice that for very narrow beams $\left(\sigma_{r} / \sigma_{z}<<1\right)$ the ratio, $k_{p 1} / k_{p 2}$ is approaches unity.

To make connection with previous results $[2,3]$ we define a new function $\Pi\left(\frac{\sigma_{r}}{\sigma_{z}}\right)=\Theta\left(\frac{\sigma_{r}}{\sigma_{z}}\right) /\left(\frac{\sigma_{r}}{\sigma_{z}}\right)$ (which is plotted in Fig.3). Using this function, we can rewrite $E_{z M}$ as:

$$
E_{z M}=\frac{q N}{\sigma_{z}^{2}} \Pi\left(\frac{\sigma_{r}}{\sigma_{z}}\right)
$$

The function $\Pi\left(\frac{\sigma_{r}}{\sigma_{z}}\right)$ is a relatively slowly changing logarithmic function. To obtain useful scaling laws this weak logarithmic dependence was neglected leading to the well known $\frac{1}{\sigma_{z}^{2}}$ scaling law cited in the literature $[2,3]$.

However, although this dependence is weak it is still significant over the range of parameters being used in present experiments and being considered in future experiments. 


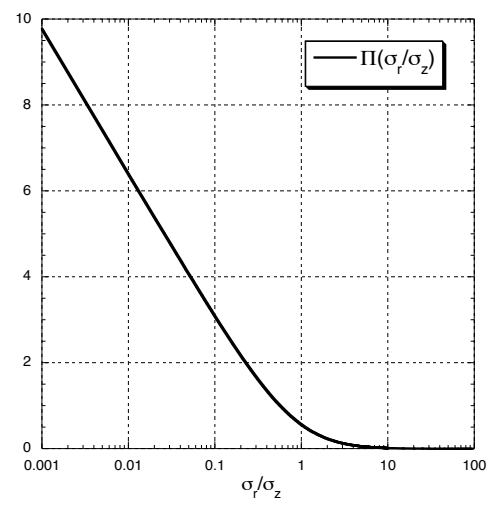

FIGURE 3. A plot of the $\Pi\left(\frac{\sigma_{r}}{\sigma_{z}}\right)=\Theta\left(\frac{\sigma_{r}}{\sigma_{z}}\right) /\left(\frac{\sigma_{r}}{\sigma_{z}}\right)$

\section{AN ENGINEERING FORMULA FOR THE WAKE AMPLITUDE}

In the limit of $\sigma_{r} / \sigma_{z}<<1$ we can obtain a very accurate asymptotic expansion for $\Pi\left(\frac{\sigma_{r}}{\sigma_{z}}\right)$. If we use the result in eq. (6) in eq. (7) and then maximize the expression with respect to $\mathrm{k}_{\mathrm{p}}{ }^{2}$, it is found that this occurs for $k_{p} \sigma_{z} / \sqrt{2}=1$. This is also seen in figure 2 where the optimized field and that for $k_{p} \sigma_{z} / \sqrt{2}=1$ are identical for small $\mathrm{r}_{\mathrm{a}}$. Using this fact, it is straightforward to obtain the asymptotic expression,

$$
\begin{aligned}
\Pi\left(\frac{\sigma_{r}}{\sigma_{z}}\right) & \approx \frac{2}{\mathbf{e}}\left\{-0.577-2 \ln \left(\frac{\sigma_{r}}{\sigma_{z}}\right)\right\} \\
& \approx \frac{4}{\mathrm{e}}\left\{0.05797-\ln \left(k_{p} \sigma_{r}\right)\right\}
\end{aligned}
$$

Therefore, in the small $r_{a}$ limit, we find:

$E_{z M}=\frac{q N}{\sigma_{z}^{2}} \Pi\left(\frac{\sigma_{r}}{\sigma_{z}}\right) \approx \frac{q N}{\sigma_{z}^{2}}\left\{\frac{4}{\mathrm{e}}\left(0.05797-\ln \left(k_{p} \sigma_{r}\right)\right)\right\}$

or in normalized units by ignoring 0.05797 for typically small $k_{p} \sigma_{r}$ : 


$$
\frac{e E_{z M}}{m c \omega_{p}} \equiv \beta \approx 1.3 \frac{q}{e} \frac{n_{b}}{n_{p}} k_{p}^{2} \sigma_{r}^{2} \ln \left(\frac{1}{k_{p} \sigma_{r}}\right)
$$

We can also rewrite this in the following form for comparisons with experiments:

$$
E_{z M} \approx(236 M V / m)\left(\frac{q}{e}\right)\left(\frac{N}{4 \times 10^{10}}\right)\left(\frac{600 \mu m}{\sigma_{z}}\right)^{2} \ln \left(\sqrt{\frac{10^{16} \mathrm{~cm}^{-3}}{n}} \frac{50 \mu m}{\sigma_{r}}\right)
$$

Here, we have ignored 0.05797 compared to $\ln 1 / k_{p} \sigma_{r}$ in eq. 13. This is true for typical $k_{p} \sigma_{r}$ values in experiments. This engineering formula is identical to eq. 1 of ref. $[2,3]$ except for the natural logarithmic term. As we have note before, the slow logarithmic term can vary by factor of four for typical experimental parameters, so comparisons to experiments need include this term. Last, we note it is not possible to simply merge the narrow and large spot size wake expressions via $\left.e E=\sqrt{n_{p}}(e V / \mathrm{cm}) \frac{n_{b}}{n_{p}} \frac{\sqrt{2 \pi} k_{p} \sigma_{z} e^{-\frac{k_{p}^{2} \sigma_{z}^{2}}{2}}}{1+\frac{1}{k_{p}^{2} \sigma_{r}^{2}}}\right) \operatorname{Sin}\left(k_{p}(z-c t)\right)$ as was done in ref [2] Such an expression does not include the natural logarithmic factor.

\section{ACKNOWLEDGMENTS}

This work is supported by the US Department of Energy through the Grant No. DEFG02-03ER54721 and DE-FG03-92-ER4727.

\section{REFERENCES}

1. T. Katsouleas et al., Particle accelerators, Vo.22 pp.81-99 (1987) and references therein

2. S.Lee,T.Katsouleas,R.Hemker, E.S.Dodd,W.B.Mori, Phys.Rev.E 64, 045501(R) (2001)

3. S.Lee et al., Phys. Rev.ST. Beams 5,011001 (2002) 